\title{
NOTES ON CONTRIBUTORS
}

Andrew Bonnell, Department of History, The University of Queensland, Brisbane, Qld 4072, Australia; e-mail: a.bonnell@mailbox.uq.edu.au David Brody, Department of History, University of California, Davis, CA 956r6, USA; e-mail: brodyiir@uclink4.berkeley.edu

Willy Buschak, CES/ETUC/EGB/DEFS, Boulevard Jacqmain I 55, I 2 Io Bruxelles, Belgium; e-mail: Nbenchay@ETUC.org

John Callaghan, Division of History, Politics and International Studies, University of Wolverhampton, Dudley Campus, Castle View, Dudley DYI 3HR, UK; e-mail: mi2940@wlv.ac.uk

Norman Caulfield, Department of History, Fort Hays State University, 600 Park Street, Hays, KS 6760I-4099, USA; e-mail: ncaulfie@fhsu.edu Michael David-Fox, Department of History, University of Maryland, 2 I I 5 Francis Scott Key Hall, College Park, MD 20742-73 I s, USA; e-mail: mdavidf@wam.umd.edu

Clive Emsley, Department of History, Faculty of Arts, The Open University, Walton Hall, Milton Keynes $\mathrm{MK}_{7}$ 6AA, UK; e-mail: c.emsley@open.ac.uk

Peter Friedemann, Institut für soziale Bewegungen, Ruhr-Universität Bochum, Clemensstrasse I7-I9, 44789 Bochum, Germany; e-mail: Peter.Friedemann@ruhr-uni-bochum.de

Venus Green, Department of History, CUNY - The City College, I38th Street at Convent Avenue, New York, NY ro03 I, USA; e-mail: NYCENSUS@aol.com

Gijs Kessler, Istituto Universitario Europeo, Villa Schifanoia, Via Boccaccio I 2 I, 50I 33 Firenze, Italy; e-mail: kessler@datacomm.iue.it

Anna Lindberg, Department of History, Lund University, P.O. Box 2074, 22002 Lund, Sweden; e-mail: anna.lindberg@hist.lu.se

Chris Lorenz, Vakgroep Geschiedenis, Faculteit der Letteren, Vrije Universiteit, De Boelelaan i I05, I08 I HV Amsterdam, The Netherlands; e-mail: lorenz@let.vu.nl

Jan Lucassen, Instituut voor Sociale Geschiedenis, Cruquiusweg 3 I, IOI9 AT Amsterdam, The Netherlands; e-mail: jlu@iisg.nl 